\title{
Artéria gastroepiplóica direita na cirurgia de revascularização do miocárdio
}

\author{
Luiz César GUARITA SOUZA*, Januário Manuel de SOUZA*, Marcos BERLINCK*, \\ Sérgio Almeida de OLIVEIRA*
}

RBCCV 44205-482

Guarita Souza L C, Souza J M, Berlinck M, Oliveira S A - Artéria gastroepiplóica direita na cirurgia de revascularização do miocárdio. Rev Bras Cir Cardiovasc 2000; 15 (1): 16-22.

RESUMO: Casuística e Métodos: Entre janeiro de 1996 e julho de 1999, a artéria gastroepiplóica direita (AGED) foi usada como enxerto na revascularização do miocárdio (RM) de 26 pacientes, sendo 25 do sexo masculino, com média de idade de 55,2 anos. Indicamos a operação em pacientes que apresentavam obstruções críticas e/ou totais nas artérias coronárias direita, interventricular anterior e ramos da artéria coronária circunflexa. Angina estável foi diagnosticada em 15 (57,8\%) pacientes e angina instável em 10 $(38,4 \%)$; 1 paciente apresentou isquemia silenciosa. Cinco (19,2\%) pacientes foram submetidos à reoperação, sendo em 2 a segunda reoperação. A circulação extracorpórea (CEC) foi usada em 24 pacientes, normotérmica em 18 (75\%) e hipotérmica em 6 (25\%). Dois, 5 e 19 pacientes apresentavam obstruções em um, dois e três vasos coronários, respectivamente. A AGED foi usada na forma pediculada em 24 pacientes, servindo de enxerto para o ramo interventricular anterior (3), artéria coronária direita (8) e seus ramos descendente (11) e ventricular posteriores (1) e ramo descendente posterior da artéria circunflexa (1). Como enxerto livre, a AGED revascularizou o ramo diagonal em 2 pacientes. Utilizamos, concomitantemente as artérias torácicas internas esquerda e direita, radial esquerda e a veia safena magna para a total RM.

Resultados: Angiografia seletiva de controle pós-operatório foi realizada em 12 (46,7\%) pacientes. Em 10 $(83,4 \%)$ visibilizou-se que a AGED estava pérvia, em 1 não foi possível cateterizar o tronco celíaco e no outro o enxerto estava ocluído. Não houve óbito hospitalar/30 dias e todos os pacientes tiveram alta hospitalar assintomáticos.

Conclusão: Concluímos que a AGED, neste estudo, mostrou ser um enxerto seguro para ser utilizado na operação de RM. irrigação.

DESCRITORES: Revascularização miocárdica, métodos. Artérias torácicas, transplante. Estômago,

\section{INTRODUÇÃO}

A revascularização miocárdica (RM) utilizando-se a veia safena magna como enxerto foi um marco na cirurgia cardíaca moderna (1), entretanto, alguns trabalhos, dentre eles o de BOURASSA et al. (2) mostraram que os enxertos venosos eram susceptíveis ao desenvolvimento de aterosclerose.
Por esta razão os enxertos arteriais passaram a ser mais valorizados e, assim, estão sendo cada vez mais usados nas operações de RM: pelos bons resultados que a artéria torácica interna esquerda apresenta quando anastomosada ao ramo interventricular anterior ${ }^{(3)}$, pelo baixo índice de desenvolvimento de aterosclerose nestes enxertos e por indícios de que os enxertos arteriais

Trabalho realizado no Serviço de Cirurgia Cardiovascular do Prof.Dr.Sérgio Almeida de Oliveira, no Hospital Beneficência Portuguesa de São Paulo, SP, Brasil.

Recebido para publicação em setembro de 1999.

* Do Hospital Beneficência Portuguesa de São Paulo.

Endereço para correspondência: Luiz César Guarita Souza. Rua Maestro Cardim, 769 - 2oa andar-Sala 233. São Paulo, SP, Brasil. CEP: $01323-001$. Tel: (011) 283-1613. 
Guarita Souza L C, Souza J M, Berlinck M, Oliveira S A - Artéria gastroepiplóica direita na cirurgia de revascularização do miocárdio. Rev Bras Cir Cardiovasc 2000; 15 (1): 16-22 .

apresentam uma maior longevidade frente aos enxertos venosos.

A busca por novos enxertos arteriais, tais como as artérias torácica interna direita, radial, gastroepiplóica, ulnar e epigástrica inferior tem sido avaliada desde então.

A artéria gastroepiplóica direita(AGED) foi usada pela primeira vez por BAILEY et al. ${ }^{(4)}$, em 1966 , experimentalmente em cães. PYM et al. (5), em 1984, revascularizaram a parede inferior do coração de 9 pacientes. No Brasil, os pioneiros desta técnica foram BUFFOLO et al. (6), em 1987. Entretanto, foi SUMA et al. (7-12) que difundiram o emprego da AGED como enxerto na RM.

O objetivo deste trabalho é analisar o comportamento da AGED como enxerto na operação de $\mathrm{RM}$, através da experiência clínica de uma série consecutiva de 26 pacientes operados.

\section{CASUÍSTICA E MÉTODOS}

Entre janeiro de 1996 e julho de 1999, foram realizadas 3.432 operações para RM. A AGED foi usada como enxerto na operação de RM em 26 $(0,75 \%)$ pacientes. O sexo masculino foi prevalente com $25(96,2 \%)$ casos. A idade variou de 33 a 71 anos, com média de 55,2 anos.

A AGED foi sempre utilizada como uma segunda ou terceira opção de enxerto arterial tendo sido usada, preferencialmente, para revascularizar as artérias coronárias direita e seus ramos descendente e ventricular posteriores, quando estes apresentavam lesões obstrutivas subtotais ou totais. Para o ramo interventricular anterior, a AGED foi usada quando a artéria torácica interna esquerda já havia sido utilizada anteriormente.

Nenhum destes pacientes havia sido submetido previamente à operação abdominal superior ou apresentava antecedentes de gastrite e/ou úlcera gastroduodenal.

Angina estável foi diagnosticada em 15 (57,8\%) pacientes, angina instável em $10(38,4 \%)$, e 1 (3,8\%) apresentava isquemia silenciosa. Dois pacientes com angina instável apresentavam concomitantemente, insuficiência cardíaca classe II e III da NYHA.

Dois $(7,7 \%)$ pacientes tinham lesão coronária obstrutiva em apenas um vaso; 5 (19,2\%) tinham lesão biarterial e em $19(73,1 \%)$ casos as lesões estavam presentes em três artérias. Um (3,8\%) destes pacientes apresentava, concomitantemente, lesão em tronco de artéria coronária esquerda.

Dos 26 pacientes operados, 21 (80,8\%) foram submetidos à operação cardíaca pela primeira vez e $5(19,2 \%)$ estavam sendo reoperados, 2 deles pela segunda vez.

A dissecção da AGED consistiu primeiramente em uma esternotomia mediana transesternal, um pouco maior que o habitual, e abertura do pericárdio.

Após identificação da bainha do músculo reto anterior do abdome abaixo do diafragma, abriu-se o peritôneo e visibilizou-se o estômago, o qual foi tracionado parcialmente para a cavidade pericárdica, através da sua grande curvatura. Na junção da grande curvatura do estômago com a inserção do omento maior identificou-se a AGED, a qual foi palpada para avaliar seu pulso e diâmetro (Figura 1). A seguir, realizou-se sucessivas ligaduras de seus ramos gástricos curtos até atingir o piloro (Figura 2). Procedeu-se à ligadura e transecção da artéria na sua porção distal, ao nível do antro gástrico, após administração via endovenosa de 5000ui de heparina. Injetou-se $10 \mathrm{ml}$ de solução de papaverina (100mg

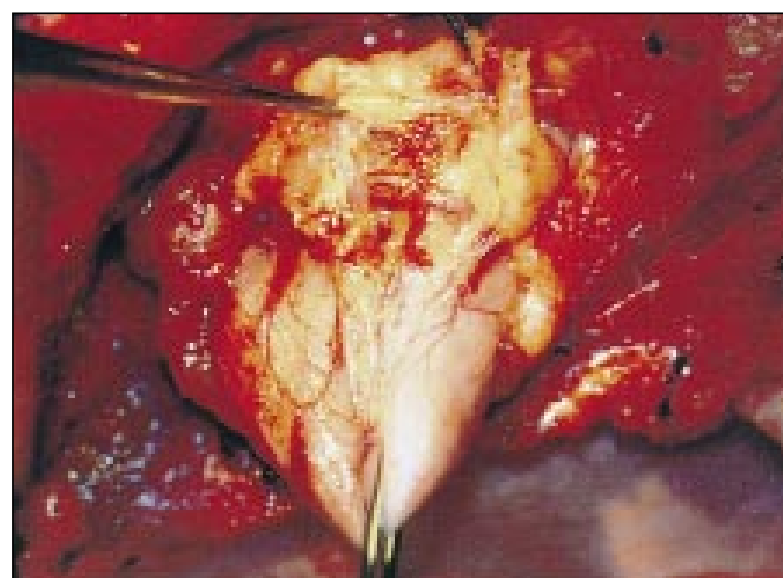

Fig. 1 - Junção do omento maior com a grande curvatura do estômago. Início da dissecção da AGED.

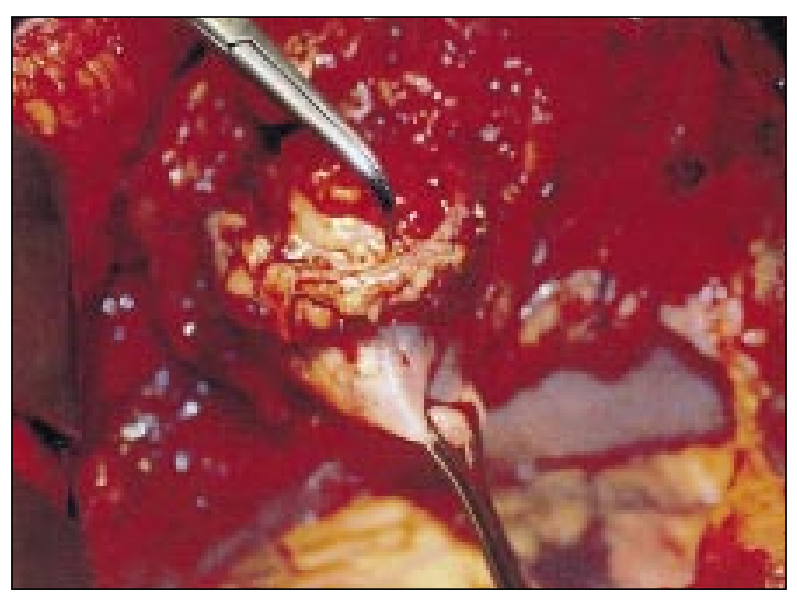

Fig. 2 - AGED dissecada, mostrando sua porção proximal, ao nível do piloro. 
Guarita Souza L C, Souza J M, Berlinck M, Oliveira S A - Artéria gastroepiplóica direita na cirurgia de revascularização do miocárdio. Rev Bras Cir Cardiovasc 2000; 15 (1): 16-22.

em $100 \mathrm{ml}$ SF $0,9 \%$ ) na luz da artéria, com o objetivo de eliminar ou evitar espasmo e aumentar seu diâmetro (Figura 3). A AGED chegou a atingir $25 \mathrm{~cm}$ de comprimento (Figura 4).

O estômago foi reposicionado na cavidade abdominal e a AGED levada ao tórax, através de um orifício no diafragma, passando pela frente do estômago e do fígado. O peritôneo parietal foi suturado sem drenar a cavidade abdominal.

A AGED serviu de enxerto para a artéria coronária direita em $8(30,9 \%)$ pacientes, para o ramo descendente posterior da artéria coronária direita em $11(42,3 \%)$, para o ramo interventricular anterior em $3(11,5 \%)$, para a artéria coronária diagonal em $2(7,7 \%)$, para o ramo descendente posterior da artéria coronária circunflexa em $1(3,8 \%)$ e para o ramo ventricular posterior da artéria coronária direita também em $1(3,8 \%)$ paciente. As anastomoses término-laterais entre a artéria gastroepiplóica direita e as artérias coronárias foram realizadas por meio de sutura contínua, com fio polipropilene monofilamentar 8.0.

Quanto ao posicionamento da AGED, optou-se pelo fluxo anterógrado para revascularizar as artérias coronárias, com exceção do ramo interventricular anterior, que foi revascularizado no contrafluxo.

Realizamos a fixação do pedículo da AGED ao epicárdio próximo à anastomose, com o objetivo de se evitar tração ou acotovelamento do mesmo.

Circulação extracorpórea(CEC) com hemodiluição e anticoagulação de $4 \mathrm{mg} / \mathrm{kg}$ de peso, através da canulação da aorta ascendente e do átrio direito, foi utilizada em 24 (92,3\%) pacientes. Em 2 (7,7\%) pacientes submetidos à reoperação não foi necessária esta assistência.

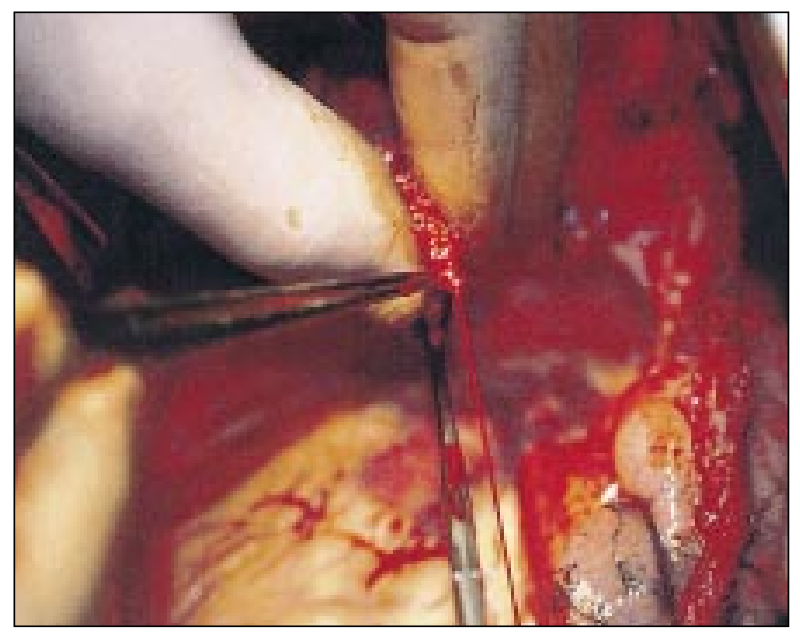

Fig. 3 - Fluxo da AGED após sua transecção.

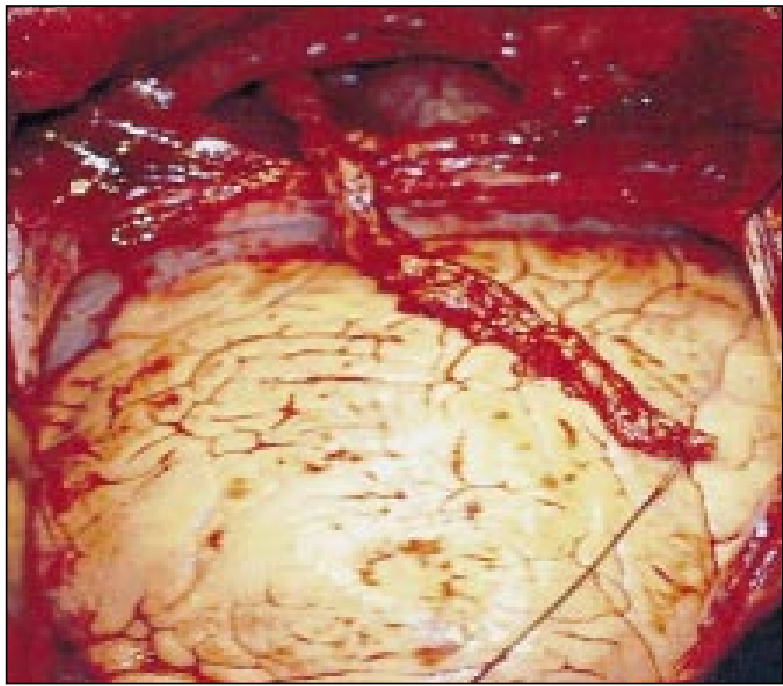

Fig. 4 - AGED totalmente dissecada, passando da cavidade abdominal para a pericárdica.

A normotermia foi utilizada em $20(76,9 \%)$ pacientes e hipotermia moderada em 6 (23,1\%). Usouse a cardioplegia sangüínea anterógrada ${ }^{(13)}$ em 23 $(88,4 \%)$ pacientes e pinçamento intermitente da aorta em $1(3,8 \%)$.

A artéria torácica interna esquerda foi usada em 21 pacientes, a direita em 8 , a artéria radial esquerda em 16 e a veia safena magna em 7 casos. Setenta e oito artérias coronárias foram revascularizadas, com média de três enxertos por paciente, sendo $91 \%$ arteriais.

A AGED foi usada na forma pediculada em 24 $(92,3 \%)$ pacientes e na forma de enxerto livre em $2(7,7 \%)$, sendo, nestes casos, para o ramo diagonal. O tempo de CEC variou de 35 a 115 minutos, média de 69,5 minutos e o tempo de pinçamento da aorta, de 25 a 77 minutos, média de 51,6 minutos.

\section{RESULTADOS}

Não houve óbito imediato - hospitalar e até 30 dias de pós-operatório - nesta série.

Não se observou, em nenhum dos pacientes submetidos à operação, infarto do miocárdio pósoperatório, entretanto, 1 (3,8\%) paciente apresentou baixo débito cardíaco e supradesnivelamento do segmento ST ao eletrocardiograma, nas derivações pré-cordiais, sugerindo isquemia na parede anterior da ventrículo esquerdo. Optou-se pela revisão cirúrgica imediata, na qual foi observada trombose da artéria torácica interna esquerda que estava anastomosada ao ramo interventricular anterior. Realizouse uma ponte de veia safena para o ramo interven- 
Guarita Souza L C, Souza J M, Berlinck M, Oliveira S A - Artéria gastroepiplóica direita na cirurgia de revascularização do miocárdio. Rev Bras Cir Cardiovasc 2000; 15 (1): 16-22 .

tricular anterior, distalmente à anastomose antiga, com regressão das alterações isquêmicas. Este paciente teve evolução satisfatória e alta hospitalar após a realização de controle angiográfico pós-operatório, o qual comprovou a oclusão da artéria torácica interna esquerda com perviabilidade dos demais enxertos, inclusive da AGED.

Dois pacientes evoluíram com derrame pleural esquerdo, 1 paciente com fibrilação atrial e outro teve infecção pulmonar. Importante relatar que não se observou nenhum episódio de sangramento abdominal após a dissecção da AGED.

A sonda nasogástrica foi retirada tão logo iniciou o peristaltismo intestinal; o que ocorreu no primeiro dia de pós-operatório.

Dos 26 pacientes operados, 12 (46,7\%) foram submetidos a estudo cineangiocoronariográfico pósoperatório da AGED, através do cateterismo seletivo do tronco celíaco, antes da alta hospitalar. Em $10(83,4 \%)$ pacientes a AGED estava pérvia, com bom fluxo para a artéria coronária (Figuras 5 e 6 ). Em 1 paciente não foi possível cateterizar seletivamente o tronco celíaco, não sendo possível sua avaliação. No outro paciente, a AGED estava ocluída.

O tempo de internação na Unidade de Terapia Intensiva variou de 36 a 60 horas, média de 39,3 horas. O tempo de internação hospitalar variou de seis a 25 dias, média de 8,8 dias.

\section{COMENTÁRIOS}

A AGED, embora utilizada há bastante tempo, somente passou a receber mais atenção com os

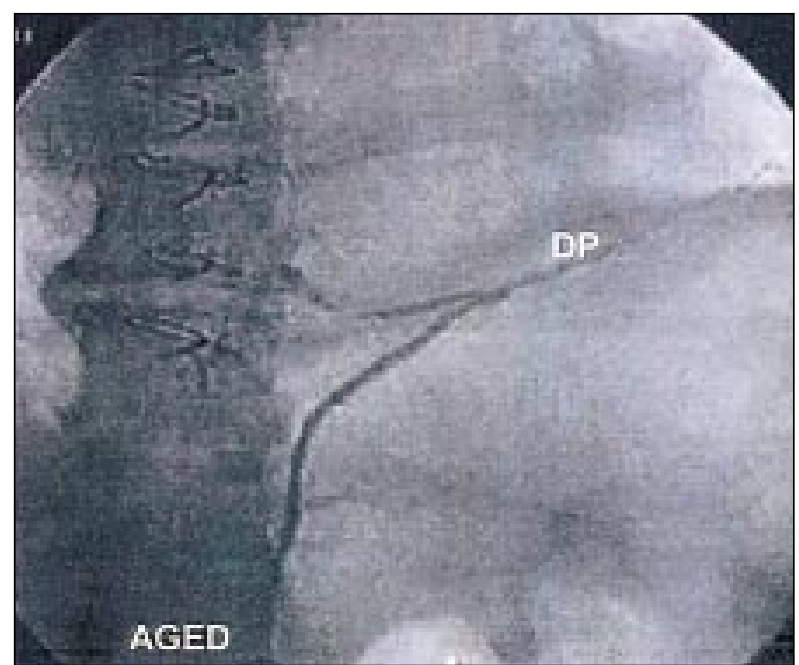

Fig. 5 - Angiografia seletiva do tronco celíaco onde visibilizamos a AGED pérvia, irrigando o ramo descendente posterior da artéria coronária direita.

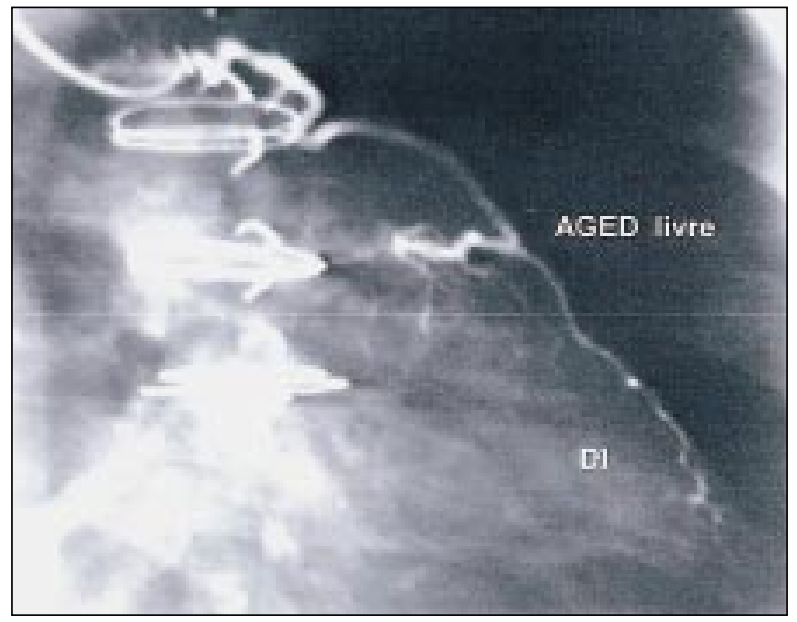

Fig. 6 - Coronariografia mostrando AGED pérvia, anastomosada proximalmente à aorta ascendente e distalmente ao ramo diagonal.

trabalhos de SUMA et al. (7-12) que mostraram altas taxas de perviabilidade pós-operatória.

$\mathrm{Na}$ seleção dos pacientes para a operação de RM utilizando a AGED como enxerto, demos preferência aos pacientes que apresentavam artérias coronárias ocluídas e/ou com lesão obstrutiva subtotal, para que não houvesse competição de fluxo entre a artéria coronária e o enxerto.

A artéria torácica interna esquerda foi utilizada como enxerto para o ramo interventricular anterior, mesmo nos casos em que ela estava ocluída, pois já está comprovado na literatura seus bons resultados a longo prazo. Utilizamos a AGED para revascularizar o ramo interventricular anterior em 3 (11,6\%) pacientes; em 2 deles eram reoperações e já haviam utilizado a artéria torácica interna esquerda na primeira RM e em 1, a artéria torácica interna esquerda foi lesada durante sua preparação. Nestes 3 pacientes, em que não pudemos utilizar a artéria torácica interna esquerda, observamos que a AGED foi uma boa alternativa para revascularizar o ramo interventricular anterior, como foi sugerido por SUMA et al. (12) e CREMER et al. (14).

O início da dissecção da AGED é bem definido quando se delimita a junção do omento maior com a grande curvatura do estômago, sendo que deve ser realizada com muito cuidado, evitando manipulação excessiva, queimadura pelo eletrocautério e hematoma de sua adventícia, pois o enxerto é muito susceptível a espasmos, os quais foram observados pelo simples toque em sua parede. Foi com base nesta situação que, logo após a sua dissecção, injetamos solução de papaverina na luz do vaso e administramos bloqueador de canal de cálcio, tanto 
via sublingual, como endovenosa e, eventualmente, nitroglicerina, na tentativa de aumentar seu diâmetro e diminuir o risco de espasmo, como foi sugerido por SUMA (9) e YANG et al. (15). À medida que realizamos as ligaduras dos ramos da AGED, observamos que o enxerto tem geralmente um tamanho satisfatório, com diâmetro entre 1,5 e 3,0 mm, como foi descrito por HIDALGO et al. (16) e MALUF et al. (17). A porção proximal, ao nível do piloro, geralmente, apresenta calibre maior, ao passo que, a sua porção distal, na altura do antro gástrico, é mais fina, sendo seu fluxo comparável ao da artéria torácica interna. Em nenhuma das AGED dissecadas à ectoscopia encontramos sinal sugestivo de aterosclerose, comprovando nossas expectativas.

A passagem da AGED da cavidade abdominal para a pericárdica pode ser feita de várias maneiras: pela frente e por trás do estômago, por dentro, pela frente e por trás do fígado, como sugerido por RAMSTROM et al. (18). Optamos por posicionar a artéria anteriormente ao estômago e ao fígado, até atingir o diafragma, evitando assim sua compressão pelas estruturas vizinhas e o estiramento excessivo, o que poderia comprometer sua perviabilidade.

Com relação à direção do fluxo da AGED, acreditamos que, tanto o anterógrado quanto o retrógrado conseguem irrigar a artéria coronária de forma semeIhante, pois o que define a direção do fluxo é a posição da artéria coronária a ser revascularizada.

Utilizamos preferencialmente a perfusão normotérmica, pois acreditamos que a hipotermia possa causar espasmo no enxerto arterial.

Tem sido evitado a indicação da AGED como enxerto na RM nos pacientes que apresentem alguma chance de serem submetidos à operação abdominal, posteriormente à operação cardíaca, uma vez que durante a manipulação abdominal superior sempre existe o risco de comprometer a viabilidade do enxerto.

Histologicamente, a AGED apresenta túnica média com predomínio de células musculares, talvez seja este fator o grande responsável pela maior susceptibilidade a espasmos. Entretanto, a baixa tendência da AGED em desenvolver aterosclerose, juntamente com os fatores de relaxamento endotelial - óxido nítrico e a prostaciclina - presentes na sua estrutura histológica, podem evitar a agregação plaquetária, sugerindo maior longevidade do enxerto.

Acreditamos que a utilização da AGED pediculada ou anastomosada diretamente à artéria torácica interna esquerda traga melhores resultados, haja vista que as pressões nos vasos são semelhantes e menores do que na aorta ascendente, poupando, desta forma, seu endotélio. Contudo, em 1 (3,8\%) caso tivemos que anastomosar a AGED diretamente à aorta ascendente porque a artéria torácica interna esquerda não apresentava diâmetro favorável.

Não foi possível realizar estudo angiográfico de controle pós-operatório em todos os pacientes operados, já que vários recusaram a indicação para novo procedimento invasivo. Entretanto, nos 12 $(46,7 \%)$ pacientes estudados, o enxerto estava pérvio em $10(83,4 \%)$. Destes, em 9, a AGED estava na forma pediculada e, em 1, na forma livre, anastomosada à artéria coronária diagonal e à aorta ascendente.

No caso em que não foi possível cateterizar a AGED não observamos alteração isquêmica no eletrocardiograma pós-operatório, e, nem elevação enzimática sugestiva de infarto do miocárdio. O paciente manteve-se assintomático até a alta hospitalar.

Pudemos realmente observar nos casos operados uma menor tendência da AGED em desenvolver a doença aterosclerótica, sendo, neste aspecto, muito semelhante à artéria torácica interna. Como a ausência de aterosclerose é um fator determinante para a longevidade do enxerto, acreditamos que a AGED poderá ter uma boa evolução.

A AGED pôde ser usada como enxerto na operação de $\mathrm{RM}$ sem aumentar o risco cirúrgico, apresentando satisfatória perviabilidade precoce. Nesta série de pacientes não observamos aumento da morbidade pós-operatória em decorrência do uso da AGED.

A taxa de $83,4 \%$ de perviabilidade da AGED observada nos pacientes que foram submetidos a controle angiográfico pós-operatório imediato foi semelhante à obtida por CREMER (14) que apresentou perviabilidade pós-operatória imediata da AGED de $80 \%$. Entretanto, SUMA et al (11) que, sem dúvida, detêm a maior casuística mundial de utilização da AGED, relatam taxas de perviabilidade imediata superiores, entre $94 \%$ e $97 \%$, dados semelhantes aos encontrados em controles pós-operatórios da artéria torácica interna esquerda quando anastomosada ao ramo interventricular anterior.

\section{CONCLUSÃO}

A AGED é um enxerto arterial seguro, de fácil preparo, com bom fluxo sangüíneo e que pode ser utilizada preferencialmente para revascularizar a face diafragmática do ventrículo esquerdo e também como uma segunda opção para revascularizar o ramo interventricular anterior.

O uso da AGED como enxerto na operação de RM ainda está buscando seu espaço no contexto da 
Guarita Souza L C, Souza J M, Berlinck M, Oliveira S A - Artéria gastroepiplóica direita na cirurgia de revascularização do miocárdio. Rev Bras Cir Cardiovasc 2000; 15 (1): 16-22.

Cardiologia atual, mas acreditamos que, com o tempo, com uma maior experiência em sua utilização, criando-se uma rotina para sua dissecção, teremos parâmetros para consagrar o seu uso como enxerto de excelência. Mas, para que isto ocorra, será preciso, realmente, comprovar a sua perviabilidade a médio e longo prazo, da mesma forma que a da artéria torácica interna esquerda foi comprovada.

RBCCV 44205-482

Guarita Souza L C, Souza J M, Berlinck M, Oliveira S A - Gastroepiploic artery in coronary artery bypass grafting. Rev Bras Cir Cardiovasc 2000; 15 (1): 16-22 .

ABSTRACT: Material and Methods: From January 1996 to July 1999, the right gastroepiploic artery (RGA) has been used for coronary artery bypass grafting (CABG) in 26 patients ( 25 male, average age 55.2 years). The surgery was indicated in patients with occlusion or critical coronary disease in the right coronary artery, in the anterior descending coronary artery and in branches of the circumflex coronary artery. Some 15 $(57.8 \%)$ patients had stable angina, $10(38.4 \%)$ had unstable angina and one of them had no clinical signs. There were $5(19.2 \%)$ reoperations, 2 of them were second reoperations. CABG was used in 24 patients; normothermic in 18 and hypothermic in six. Two, 5 and 19 patients had coronary artery disease in one, two and three vessels respectively. RGA was used "in situ" in 24 patients and as a graft in 3 to the anterior descending coronary artery, 11 to the right posterior descending coronary artery, 8 to the right coronary artery, 1 to the right posterior ventricular coronary artery and 1 to the descending posterior vessel of the circumflex coronary artery and as a free graft revascularized the coronary diagonal in 2. The left and the right internal thoracic artery, the left radial artery and the saphenous vein graft were used concomitantly.

Results: Postoperative angiography was performed in 12 (46.7\%) patients, in 10 (83.4\%) of them the RGA was patent, in 1 it was impossible to catheterize the celiac trunk, hence the flow could not be evaluated and in the other patient the graft was occluded. There were no deaths and all patients had an excellent outcome.

Conclusion: The RGA is a safe graft for CABG.

DESCRIPTORS: Myocardial revascularization, methods. Thoracic arteries, transplantation. Stomach, blood supply.

\section{REFERÊNCIAS BIBLIOGRÁFICAS}

1 Favaloro R G - Saphenous vein autograft replacement of severe segmental coronary artery occlusion: operative technique. Ann Thorac Surg 1968; 5: 334-9.

2 Bourassa M G, Campeau L, Lesperance J, Solymoss B C - Atherosclerosis after coronary artery bypass surgery: results of recent studies and recommendations regarding prevention. Cardiology 1986; 73: 259-68.

3 Loop F D, Lytle B W, Cosgrove D M et al. - Influence of the internal-mammary -artery graft on 10-year survival and other cardiac events. $N$ Engl $\mathrm{J}$ Med 1986; 314: 1-6.

4 Bailey C P, Hirose T, Brancato R, Aventura A, Yamamoto $\mathrm{N}$-Revascularization of the posterior (diaphragmatic) portion of the heart. Ann Thorac Surg 1966; 2: 791805.

5 Pym J, Brown P M, Charrette E J, Parker J O, West R O - Gastroepiploic-coronary anastomosis: a viable alternative bypass graft. J Thorac Cardiovasc Surg 1987; 94: 256-9.

6 Buffolo E, Maluf M, Barone B, Andrade J C, Galluci C -
Revascularização direta do miocárdio através da artéria gastro-omental esquerda: uma nova alternativa de desvio aorta-coronário. Relato de caso. Arq Bras Cardiol 1987; 48: 167-71.

7 Suma $\mathrm{H}$, Fukunoto $\mathrm{H}$, Takeuchi $\mathrm{A}$ - Coronary artery bypass grafting by utilizing in situ right gastroepiploic artery: basic study and clinical application. Ann Thorac Surg 1987; 44: 394-7.

8 Suma H, Takeuchi A, Hirota $\mathrm{Y}$ - Myocardial revascularization with combined arterial grafts utilizing the internal mammary and the gastroepiploic arteries. Ann Thorac Surg 1989; 47: 712-5.

9 Suma $\mathrm{H}$ - Spasm of the gastroepiploic artery graft. Ann Thorac Surg 1990; 49: 168-9.

10 Suma H, Wanibuchi Y, Furuta, S, Takeuchi A - Does use of gastroepiploic artery graft increase surgical risk? J Thorac Cardiovasc Surg 1991; 101: 121-5.

11 Suma H, Wanibuchi Y, Terada Y, Fukuda S, Takayama T, Furuta S - The right gastroepiploic artery graft: clinical and angiographic midterm results in 200 patients. $J$ Thorac Cardiovasc Surg 1993; 105: 615-23. 
12 Suma H, Amano A, Fukuda S et al. - Gastroepiploic artery graft for anterior descending coronary artery bypass. Ann Thorac Surg 1994; 57: 925-7.

13 Buckberg G D - Hazard's of administering blood cardioplegic solution directly into the heart: cardioplegic solution are not equal. J Thorac Cardiovasc Surg 1996; 111: 283-4.

14 Cremer J, Liesmann T, Wimmer-Greinecker G, Abraham $C$, Mügge A, Haverich A - In vivo comparison of free coronary grafts using the inferior epigastric (IEA), the gastroepiploic (GEA) and the internal thoracic artery (ITA). Eur J Cardiothorac Surg 1994; 8: 240-6.

15 Yang Z, Siebenmann R, Studer M, Egloff L, Lüscher T $\mathrm{F}$ - Similar endothelium-dependent relaxation, but enhanced contratility, of the right gastroepiploic artery as compared with the internal mammary artery. $J$ Thorac Cardiovasc Surg 1992; 104: 459-64.
16 Hidalgo A L L, Fernandez R D, Hidalgo M A V, Diaz J H, Calvo M I P - Revascularización miocárdica directa com arteria gastroepiploica: una alternativa cirúrgica. Medicentro 1990; 6: 192-4.

17 Maluf M A, Buffolo E, Barone B, Andrade J C S, Gallucci C - Revascularização direta do miocárdio com artéria gastro-omental esquerda: estudo anatômico e histológico e relato de caso. Rev Bras Cir Cardiovasc 1987; 2: 159-70.

18 Ramstrom J, Jaramillo A, Cadavid E, Thuren J, Henze A - A new intraabdominal artery: the pedicled right gastroepiploic artery for myocardial revascularization. Eur J Surg 1992; 158: 25-8.

19 Guarita Souza L C - Artéria gastroepiplóica direita na cirurgia de revascularização do miocárdio. [Tese de Mestrado]. São Paulo: Faculdade de Ciências Médicas da Santa Casa de São Paulo, 1999. 77p. 